\title{
Manejo del escurrimiento de aguas pluviales desde la perspectiva de los servicios ecosistémicos. Análisis de su abordaje en ciudades capitales de la Argentina
}

\author{
Management of stormwater runoff from the perspective of \\ ecosystem services. Analysis of its implementation in the main \\ cities of Argentina
}

\author{
Paula Andrea Zapperi ${ }^{1}$ \\ Universidad Nacional del Sur-CONICET, Argentina \\ Anabella Montico ${ }^{2}$ \\ Universidad Nacional del Sur, Argentina
}

\begin{abstract}
Resumen
La incorporación del enfoque de los servicios ecosistémicos, en la planificación de los espacios urbanos, contribuye con la mitigación el impacto hidrológico de la urbanización. Bajo esta premisa, el objetivo del trabajo fue analizar las reglamentaciones asociadas al manejo de aguas pluviales en la capital nacional y las capitales provinciales de la Argentina y reconocer así, antecedentes en la implementación de políticas, que favorezcan la provisión del servicio ecosistémico de regulación hídrica. Para ello, se revisaron de ordenanzas y códigos de zonificación. Los resultados obtenidos se agruparon en: indicadores urbanísticos, zonificaciones y medidas de infraestructura verde. Si bien, el $87,5 \%$ de las ciudades analizadas presenta algún tipo de medida urbanística, relacionada con el servicio ecosistémico de regulación hídrica en la mayoría de los casos, no existe una mención explícita. Esto dificulta la cuantificación, su nivel de provisión servicio y, por lo tanto, su incorporación efectiva en los instrumentos de planificación.
\end{abstract}

Palabras clave: planificación urbana; regulación hídrica; gestión de aguas pluviales; servicios ecosistémicos; Argentina.

1 Doctora en Geografía. Investigadora Asistente en el Consejo Nacional de Investigaciones Científicas y Técnicas. Docente en el Departamento de Geografía y Turismo de la Universidad Nacional del Sur, Argentina. Correo electrónico: paula.zapperi@uns.edu.ar (D) https://orcid.org/0000-0003-0783-1467

2 Licenciada en Ciencias Ambientales. Departamento de Química, Universidad Nacional del Sur, Argentina. Correo electrónico: anabella.montico@gmail.com. (D) https://orcid.org/0000-0002-4618-2992 


\begin{abstract}
The inclusion of ecosystem services in the planning of urban spaces contributes to mitigating the hydrological impact of urbanization. Under this premise, the objective of the present work was to analyze the regulations associated with the management of stormwater in the capital and provincial capital cities of Argentina, and thus recognize background information regarding the implementation of policies that favor the provision of ecosystem services relevant to the regulation of water resources. Accordingly, zoning ordinances and codes were reviewed. The results obtained were grouped into: urban indicators, zones and green infrastructure measures. Although $87.5 \%$ of the considered cities exhibit some type of rule related to ecosystem services pertaining to the regulation of water resources, there is no explicit mention. The above situation makes it difficult to quantify the level of provision of ecosystem services and, therefore, hampers its effective inclusion in urban planning instruments.
\end{abstract}

Keywords: Urban planning; Water resource regulation; Stormwater management; Ecosystem services; Argentina.

\title{
Introducción
}

Los patrones de asentamiento urbano han sufrido una transformación significativa en las últimas décadas, pasando del auge de las grandes aglomeraciones urbanas, hacia el desarrollo de las ciudades intermedias. Estos centros, se consolidan como urbes de crecimiento acelerado y disperso, que tiende a dificultar la equidad y sostenibilidad del entorno urbano. Las implicancias ambientales de este tipo de crecimiento, se manifiestan en un aumento en la huella urbana, incrementando así, el uso intensivo de recursos naturales como el suelo, el agua y la energía.

En contraposición, se presenta el modelo de ciudades sostenibles que busca una trama urbana de cierta compacidad, que contribuya con la cercanía equitativa a todos los servicios, incluyendo las áreas verdes y el espacio público (Terraza, Rubio \& Vera, 2016). Estos nuevos modelos de urbanismo, consideran a las ciudades como ecosistemas urbanos e incorporan conceptos de sostenibilidad dentro de los criterios de la planificación territorial (Ahern, Cilliers \& Niemelä, 2014).

Se contribuye de esta manera, con el aprovechamiento de los servicios ecosistémicos disponibles y la adaptación de las urbes, al entorno en el cual se desarrollan. La tendencia hacia la integración de la planificación urbana, ecosistema urbano y ciudad sostenible puede evidenciarse en propuestas y declaraciones de organismos internacionales como Naciones Unidas. En 2015, la Asamblea General de la ONU presentó los objetivos de la Agenda 2030 para el Desarrollo Sostenible, cuyos puntos 
establecen el carácter fundamental de la gestión y el desarrollo sostenible del medio urbano, para la calidad de vida de los pueblos (Organización de Naciones Unidas, 2015).

En esta línea, el gobierno argentino a través del Ministerio de Ambiente y Desarrollo Sustentable conformó en el año 2016, la Unidad de Ciudades Sustentables (Resolución N ${ }^{\circ} 378 / 16$ ) con el objetivo de apoyar a los municipios en el desarrollo de planes de sustentabilidad con herramientas de planificación y de capacitación técnica-ambiental.

La adaptación al Cambio Climático se plantea como una de las áreas prioritarias de intervención y en virtud de ello, se respalda a la Red de $\mathrm{Mu}$ nicipios Argentinos frente al Cambio Climático (RAMCC) conformada en el año 2010 (Secretaría de Ambiente y Desarrollo Sustentable, 2019). Paralelamente, desde el Gabinete Nacional de Cambio Climático se trabaja en la Plan Nacional de Adaptación y Mitigación al Cambio Climático, de acuerdo a lo que establece la Ley $\mathrm{N}^{\circ} 27520 / 19$.

El aumento de los eventos climáticos extremos es uno de los efectos adversos, que el Cambio Climático impone a las ciudades. En la Argentina, la ocurrencia de eventos extremos de precipitación (Barros et al., 2015) se conjuga con el avance de la urbanización de llanuras de inundación, dando lugar al aumento de las inundaciones urbanas ribereñas. Por otra parte, las superficies pavimentadas y las edificaciones reducen la capacidad de la infiltración del suelo, con el consecuente aumento y aceleración de la escorrentía superficial que se concentra en calles y avenidas (González, Torchia \& Viand, 2015). Esto contribuye con el anegamiento de áreas, donde se supera la capacidad de conducción del sistema de drenaje urbano y de sectores donde el agua no logra encauzarse, debido a la topografía del terreno, ya sea por sus características naturales o por las modificaciones introducidas.

Lo mencionado, forma parte de una realidad urbana que muestra la necesidad de gestionar las ciudades, a través de un enfoque que permita la integración de consideraciones ambientales en la planificación y diseño y que permita a la vez, estimular las transformaciones necesarias de las políticas, los mercados y las conductas. En este marco, la hipótesis establecida sugiere que abordar la gestión del drenaje urbano, a través de la perspectiva de los servicios ecosistémicos puede contribuir con la generación de medidas de planificación, que incorporen aspectos propios de la dinámica hídrica natural. 
Por ello, el objetivo del trabajo fue analizar las reglamentaciones asociadas al manejo de aguas pluviales en las capitales provinciales de la Argentina. De esta manera, se buscó reconocer casos que puedan constituir un antecedente en la implementación de políticas, que favorezcan la provisión del servicio ecosistémico de regulación hídrica. A su vez, el análisis buscó determinar cuáles debieran ser los lineamientos a seguir, para la incorporación del enfoque ecosistémico en la gestión del drenaje urbano.

\section{Marco teórico}

\section{Servicios ecosistémicos en el entorno urbano}

Los servicios ecosistémicos se definen como los beneficios tangibles e intangibles, que los diferentes ecosistemas proporcionan a la sociedad de manera natural y que actúan como contribuciones directas e indirectas al bienestar humano (Gómez-Baggethun et al., 2014). Al hablar de servicios ecosistémicos, se enfatiza la interdependencia que existe entre sistemas ecológicos y sistemas sociales (Daily, 1997; Balvanera et al., 2012). Esta interacción es especialmente importante en el contexto urbano donde la infraestructura construida, cubre gran proporción de la superficie y la densidad poblacional es alta (Pickett et al., 2001).

A nivel de política, los ecosistemas urbanos a menudo se describen como "infraestructura verde", pues esta representación captura el papel que el suelo y la vegetación desempeñan en la prestación de servicios ecosistémicos, a diferentes escalas espaciales (edificio, calle, barrio, región). Sin embargo, los ecosistemas urbanos implican un concepto más amplio, pues incluye áreas de bosques, ríos o lagos impulsados por la comunidad dentro o cerca de la ciudad. Así como también, a los jardines privados que no están sujetos directamente a la planificación urbana pública (GómezBaggethn \& Barton, 2013). De esta manera, los espacios verdes y azules de las áreas urbanas (parques, patios y jardines, parcelas urbanas, humedales, ríos, lagos y estanques) constituyen ecosistemas urbanos, que prestan servicios con impacto directo en la salud y la seguridad como purificación de aire, reducción de ruido, regulación de la temperatura y mitigación de la escorrentía (Bolund \& Hunhammar, 1999).

Dado que muchos flujos ecológicos se extienden más allá de las fronteras urbanas, los ecosistemas urbanos se asocian a las áreas afectadas por los flujos de energía y materiales del núcleo urbano y suburbano, 
incluidas cuencas urbanas, bosques periurbanos y campos cultivados. Sin embargo, debido al alto grado de modificación y fragmentación, su análisis también incluye componentes específicos vinculados a la prestación de servicios como árboles individuales, superficies de agua y superficies de suelo (Gómez-Baggethun \& Barton, 2013).

\section{Clasificación según los beneficios para las ciudades}

Las funciones ecosistémicas son las que dan origen a los servicios, cuando éstos significan un beneficio para la sociedad. Por lo que, el reconocimiento de una propiedad ecosistémica como servicio es contextual y depende del beneficio humano al cual contribuye. Por otra parte, una misma función ecosistémica puede proveer más de un servicio a la vez, que un servicio puede asociarse a más de un beneficio y viceversa (Altesor et al., 2011). En la construcción de la resiliencia en las ciudades se presentan ciertos servicios ecosistémicos, que son fundamentales. Se trata de la reducción de la isla de calor, el suministro de agua, la mitigación de la escorrentía y la producción de alimentos.

Desde la Ecología Urbana, se realiza la distinción entre ecología "en" las ciudades y ecología "de" las ciudades, para enfatizar la importancia de incluir los servicios de los ecosistemas, generados tanto dentro como fuera de los límites de la ciudad. En el primer caso, se hace referencia a la generación de entornos urbanos verdes saludables y funcionales, al diseño de edificios energéticamente eficientes y a una logística sostenible.

Mientras que, por ecología "de" las ciudades se reconoce la total dependencia que tienen las ciudades del paisaje circundante y el constante "ida y vuelta" entre lo urbano y lo rural (Jansson, 2013).

La clasificación y tipificación de los servicios ecosistémicos difiere según los distintos autores, que conforman la literatura referida al tema. Aun así, existe un consenso generalizado en considerar a la Evaluación de Ecosistemas del Milenio (Millennium Ecosystem Assessment, 2005) como la clasificación de referencia para el análisis de conexiones entre el funcionamiento de la naturaleza y el bienestar y supervivencia de los seres humanos, en un contexto urbano sostenible (Jansson, 2013).

Esta evaluación es resultado de una iniciativa de múltiples organismos internacionales, que reconoce: (i) servicios de provisión (alimento, madera, agua, recursos genéticos), (ii) servicios de regulación (regulación del clima y efecto invernadero, regulación del agua y de las enfermedades), 
(iii) servicios de soporte (ciclado de nutrientes, formación de suelo, ciclo hidrológico, producción primaria, polinización y control biológico) y (iiii) servicios culturales (fuente de inspiración, recreación y estéticos).

\section{Servicio ecosistémico de regulación hídrica en el ambiente construido}

La impermeabilización que implica el crecimiento de las ciudades se traduce en la pérdida de la capacidad de infiltración del suelo y en algunos casos, de la vegetación que intercepta el agua de la lluvia. Estas funciones son propias del servicio de regulación hídrica y mitigación de la escorrentía y se asocian con la capacidad de los ecosistemas de almacenar y liberar los flujos de agua, de manera controlada. La regulación hídrica se refiere entonces a la proporción de las precipitaciones, que puede ser interceptada e infiltrada, contribuyendo con la reducción de la escorrentía superficial (Jullian, Nahuelhual, Mazzorana, \& Aguayo, 2018).

El servicio ecosistémico de regulación hídrica, se sustenta en procesos que se corresponden con los distintos subsistemas del ciclo hidrológico. De esta manera, comienza con la intercepción de la precipitación incidente por parte de la vegetación, donde una gran proporción se pierde por evaporación. El agua residual de esta etapa, fluye acoplándose a la precipitación directa, la cual llega directamente al suelo, donde una proporción fluye como escurrimiento superficial, otra se infiltra y se almacena en el suelo, quedando disponible para las plantas y el resto, se acumula en napas subterráneas. Es así que, la regulación y almacenamiento de agua dependerá de estos procesos circunscritos al balance hídrico (Figura 1), donde el tipo de cobertura y las características físicas del suelo condicionan esta función de regulación (Grizzetti , Lanzanova, Liquete, Reynaud, \& Cardoso, 2016). Así, la capacidad de infiltración del suelo o el porcentaje del sellado de la superficie, pueden ser indicadores para la cuantificación de la percolación y por ende, del servicio de regulación y mitigación de la escorrentía (Villarreal \& Bengtsson, 2005).

En el ámbito urbano, la impermeabilización sumada a la frecuente compactación de los suelos expuestos, limitan cuantiosamente la infiltración (Argañaraz \& Lorenz, 2010), aumentando el volumen de escorrentía de aguas superficiales y así, la vulnerabilidad a las inundaciones (Villarreal \& Bengtsson, 2005). Las zonas vegetadas tienen una capacidad de infiltración de aguas de lluvia entre un $85 \%$ y $95 \%$ mientras que, las zonas urbanas carentes de vegetación, que manejan sus aguas de lluvia a través 
Figura 1. Factores determinantes del servicio ecosistémico de regulación hídrica

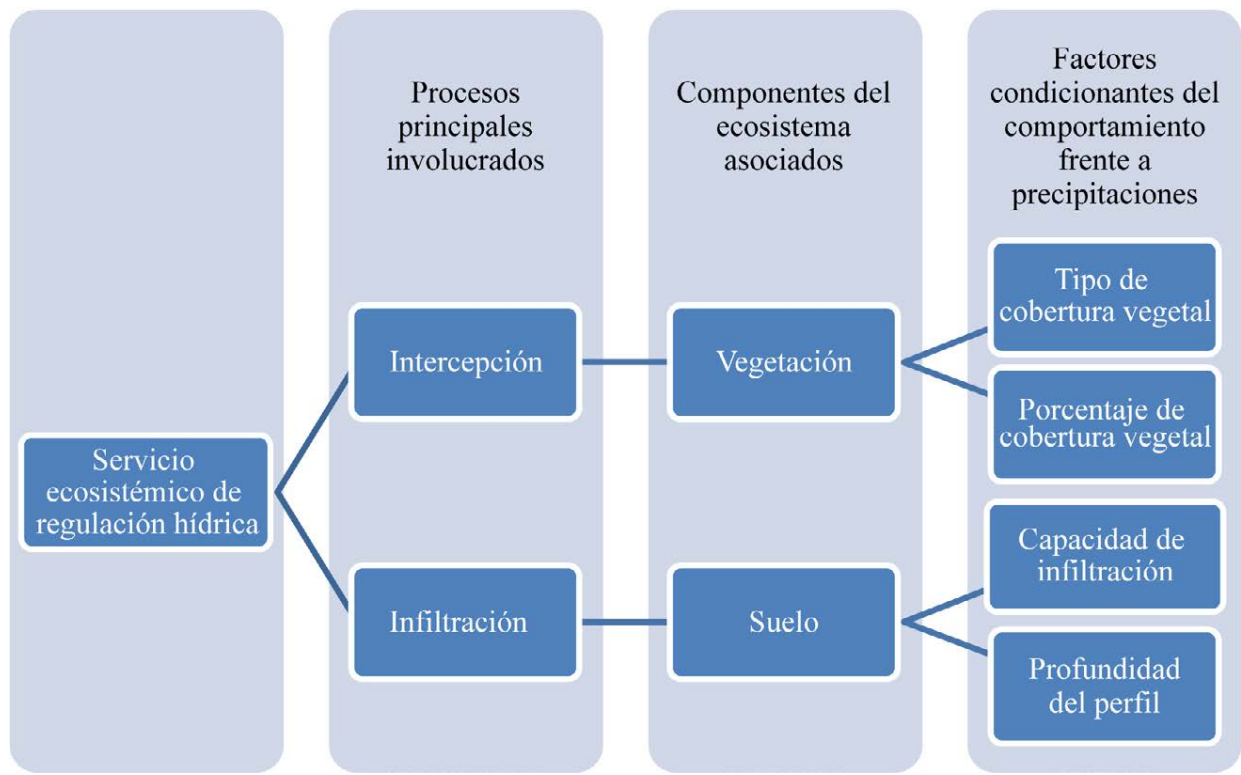

Fuente:Elaboración propia sobre la base de Grizzetti et al. (2016) y Jullian et al. (2018).

de desagües y alcantarillas, logran un nivel de infiltración de apenas el 10\% (Bernatzky, 1983 en Bolund \& Hunhammar, 1999). En este contexto, adquieren gran importancia las áreas cuyos suelos están expuestos, como es el caso de las áreas verdes y jardines domésticos, al actuar como focos de infiltración (Argañaraz \& Lorenz, 2010).

En los últimos años, se ha intensificado el interés en revertir y mitigar el impacto negativo del ambiente construido sobre el funcionamiento natural de los ecosistemas de las ciudades. Es por ello, que se busca la restauración de los servicios ecosistémicos, a través de la reproducción de sus funciones por parte de las nuevas estructuras que se construyen. Sin embargo, tal como señala Pedersen (2012), hay determinados servicios que pueden imitarse más fácilmente que otros. Servicios relacionados con la mitigación de la escorrentía y el suministro de agua, tienen un alto potencial de integración al entorno construido, entendiendo a este último como el conjunto de edificios, carreteras, infraestructura y parques que conforman el área urbana. Ejemplo de ellos son las estructuras 
para recolección y almacenamiento de agua de lluvia, el reciclaje de agua gris, las superficies con pavimentación porosa y el paisajismo que promueve el uso eficiente del agua.

\section{Hacia la incorporación de los procesos biogeofísicos en los marcos regulatorios urbanos}

La pérdida de ecosistemas en las ciudades, puede implicar altos costos económicos, a largo plazo derivados de la necesidad de restaurar y mantener los servicios públicos, a través de una infraestructura construida que equipara a los servicios provistos por la infraestructura verde urbana. Asimismo, se reconocen impactos asociados con la pérdida de resiliencia de las ciudades, hecho que aumenta su vulnerabilidad frente a distintos escenarios de riesgo (Gómez-Baggethun \& Barton, 2013).

De esta manera, el desafío de la urbanización sostenible requiere una reconexión del desarrollo humano con la capacidad de la biosfera y los servicios ecosistémicos esenciales. Dado que, la mayoría de la población mundial vive en áreas urbanas, una forma de facilitar esta reconexión, es a través de la óptica de los servicios de los ecosistemas considerando la ecología "en" y "de" las ciudades (Jansson, 2013). En los últimos años, se ha sumado el concepto de ecología "para" las ciudades como referencia a la aplicación de este enfoque, según las proposiciones normativas de cada sociedad (Tan et al., 2020).

La Evaluación de Ecosistemas del Milenio (Millennium Ecosystem Assessment, 2005) concluye que, las políticas futuras deben apuntar a satisfacer las necesidades humanas, pero a un costo menor para los sistemas naturales. Ello implica corregir el sesgo histórico en contra de la naturaleza, en la relación costo beneficio de las elecciones económicas individuales. A su vez, esta distorsión se ve agravada, pues tradicionalmente las formas de medir la riqueza no consideran el capital natural.

Paralelamente, en los ecosistemas urbanos la realización plena de sus servicios, implica la combinación del capital natural con otras formas de capital. Por ejemplo, la construcción y mantenimiento de un parque urbano, requiere no sólo del soporte natural sino también del aporte de capital financiero y de capital humano. Mientras que, los techos y paredes verdes utilizan cantidades sustanciales de materiales de construcción (celdas de drenaje, geotextiles y estructuras etc.). 
De esta manera, se establece un circuito de retroalimentación entre los distintos capitales, sobre el cual influyen las políticas públicas, las prácticas profesionales y también las costumbres sociales (Tan et al., 2020). De esta manera, la incorporación del enfoque de los servicios ecosistémicos en la planificación de los espacios urbanos, se convierte en un tema de estudio fundamental para avanzar hacia un marco regulatorio que propicie la integración de procesos biofísicos en las estructuras construidas y de esta manera, aminorar el impacto hidrológico de la urbanización. Aspecto que se presenta como una prioridad en la gestión de gran parte de las ciudades argentinas.

\section{Marco metodológico}

La potestad reguladora en materia urbanística, que han adquirido los municipios argentinos con la reforma de la Constitución de 1994, implica una ventaja para la implementación del enfoque de servicios ecosistémicos, adaptado a nivel local. De esta manera, en un primer término se realizó una revisión exploratoria de ordenanzas y códigos de zonificación, correspondientes a las ciudades capitales de las veintitrés provincias de la República Argentina y a la capital nacional (Figura 2).

Los criterios que guiaron el análisis (Figura 3) se orientaron, por un lado, a la identificación de componentes del ecosistema urbano, tales como áreas verdes, áreas azules y componentes aislados (árboles, superficies de infiltración, etc.) según las distintas escalas espaciales que quedan comprendidas en la planificación local.

En este sentido, se relevaron referencias a la generación espacios verdes y azules a escala de áreas urbanas a la vez que, se consideraron las reglamentaciones orientadas a la forestación de calles y parcelas, sean estas últimas de dominio público o de dominio privado. Precisamente, Maragno et al. (2018) detectaron que los espacios privados generan más escorrentía que los públicos, lo que evidencia la necesidad de reglar sobre los mismos. 
Paula Andrea Zapperi - Anabella Montico Management of stormwater runoff from the perspective of ecosystem services. Analysis of its implementation in the main cities of Argentina

\section{Figura 2. Distribución de las ciudades capitales de la República Argentina}

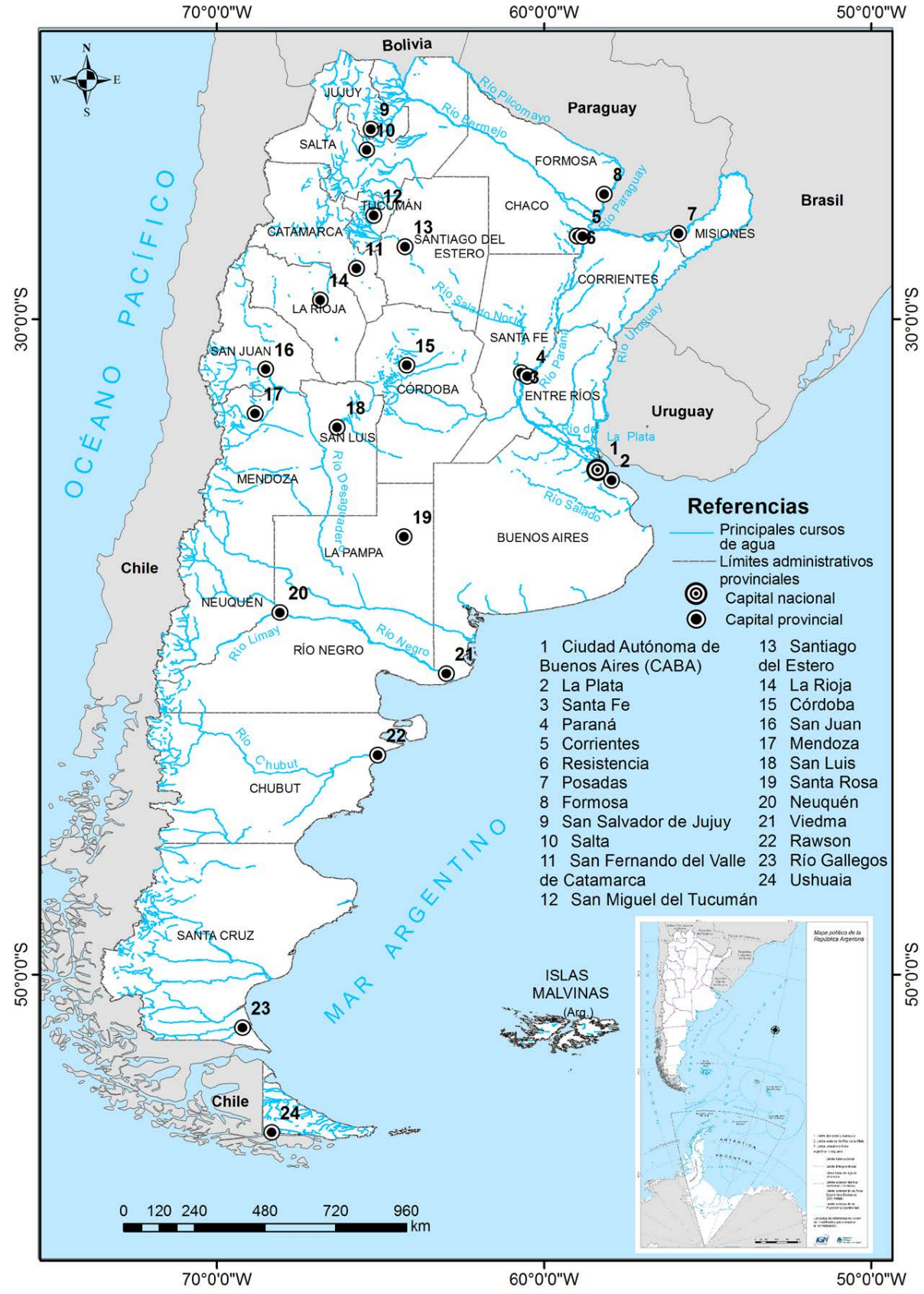

Fuente: Instituto Geográfico Nacional (IGN) de Argentina. 
Paula Andrea Zapperi - Anabella Montico

Manejo del escurrimiento de aguas pluviales desde la perspectiva de los servicios ecosistémicos. Análisis de su abordaje en ciudades capitales de la Argentina

Figura 3. Variables de análisis para la identificación de regulaciones que favorezcan al servicio ecosistémico de regulación hídrica

\section{Documentos analizados}

Escala de

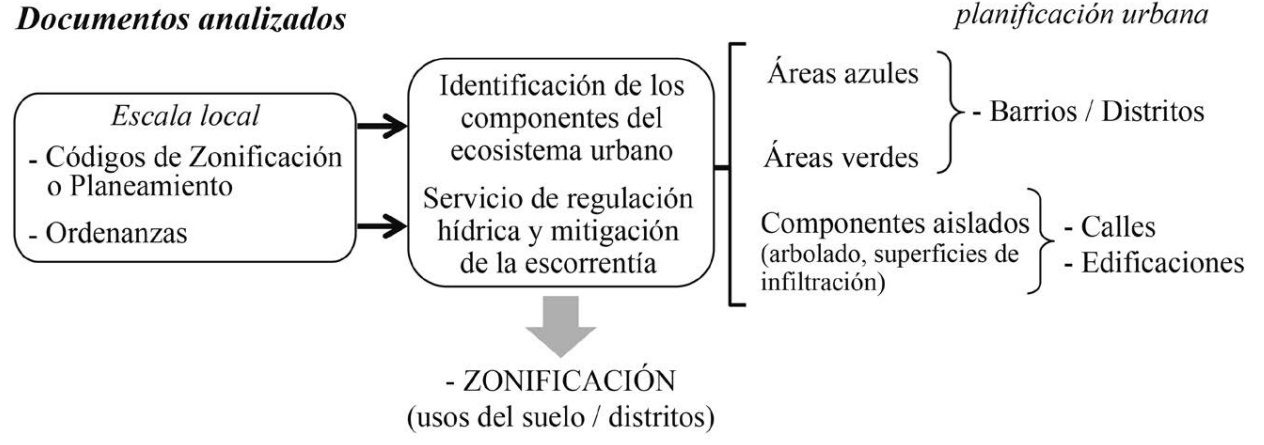

- MEDIDAS DE INFRAESTRUCTURA VERDE

- INDICADORES URBANÍSTICOS

(\% impermeabilización, FOS, FOT, etc.)

Fuente: Elaboración propia.

Por otra parte, el criterio de análisis también se orientó a reconocer la existencia de reglamentaciones que busquen la recuperación o reproducción del servicio de regulación hídrica, ya sea a través del establecimiento de determinados usos del suelo como también de la regulación de construcciones o tipos coberturas.

A su vez, para facilitar la comparación de los hallazgos en las distintas ciudades estas intervenciones, indicadores y propuestas se agruparon en: i) indicadores urbanísticos que regulen principalmente cuestiones de cobertura y capacidad constructiva dentro de las parcelas, ii) zonificación (usos del suelo), iii) intervenciones del espacio urbano, a través de la implementación de coberturas porosas e infraestructura verde.

Cabe señalar, que dentro del análisis de los casos en los que se identificó efectivamente normativa orientada a reglamentar este tipo indicadores, zonificaciones e intervenciones se buscó reconocer si en el objetivo final de su implementación, hay referencia explícita al enfoque de los servicios ecosistémicos en el ámbito urbano. 


\section{Resultados}

El relevamiento realizado permitió identificar que 21 de las 24 ciudades analizadas, presentan algún tipo de medida urbanística que se relaciona con los factores asociados al servicio ecosistémico de regulación hídrica o con la gestión de aguas pluviales, desde una perspectiva distinta a la convencional.

Excepción de ellos, son las ciudades de Rawson (provincia de Chubut), Río Gallegos (provincia de Santa Cruz) y San Miguel de Tucumán (provincia de Tucumán), que no presentan actualmente normativa al respecto. En cuanto a la mención explícita a las funciones y servicios ecosistémicos o ambientales del área a la que se refieren, sólo se halló en el caso de dos distritos de zonificación de las ciudades de Formosa y Mendoza.

Sin embargo, se identificaron conceptualizaciones incluidas dentro de las ordenanzas y códigos, que pueden ser consideradas como un puntapié inicial para la incorporación de este enfoque, en los instrumentos de planificación urbana. Tal es el caso de la Ordenanza Municipal No 9668/18 de la ciudad de Paraná (provincia de Entre Ríos), que en su artículo $2^{\circ}$ reconoce al ecosistema como un bien común, referido a "todo lo vivo y al soporte físico natural con sus componentes y elementos, de lo cual depende la subsistencia de todos los seres vivos, constituyéndolo en un derecho innegable, propiedad de todos en general y de nadie en particular". Además, conceptualiza al ambiente como una "variable restrictiva en relación a los usos del suelo y los impactos socio-ambientales".

\section{Revalorización de áreas verdes y azules a través de la zonificación}

En la implementación y generación de zonificaciones, se identifica con mayor claridad el interés por "amortiguar" el escurrimiento del agua de las lluvias y en segundo lugar, por reconstruir el equilibro ecológico en espacios fluviales. De esta manera y tal como se observa en el detalle de la tabla 1, las zonificaciones privilegian áreas ribereñas y lagunares para su preservación.

Se encuentra que estas medidas, ponen énfasis sobre los componentes del sistema urbano por sobre los servicios en sí con hincapié, por lo tanto, en áreas verdes y azules. Esto lleva a que, dentro de las ciudades identificadas, se destaquen ciudades del noreste argentino ubicadas en importantes cuencas hidrográficas o que se extienden sobre sistemas de 
lagunas. Si bien cabe hacer la salvedad de la ciudad de Mendoza, ubicada al pie de la Cordillera de los Andes.

Por otra parte, la ubicación y denominaciones de las zonificaciones establecidas indican que, en muchos casos se busca la mitigación de los efectos de las lluvias y crecidas. De este modo, la regulación hídrica entra en el esquema, pero como función ecosistémica dentro del servicio de amortiguación de las inundaciones. En este marco, se puede citar el ejemplo del "Área de Seguridad Hídrica" delimitada en la ciudad de Mendoza (OM N³944/18) para valorizar la vegetación en las zonas donde se conectan los distintos cauces que se activan durante las precipitaciones y que contribuyen con el peligro aluvional.

En cuanto a la ocurrencia de inundaciones, la ciudad de Santa Fe se destaca por las diversas zonificaciones que se desprenden de los distintos grados de protección ante las crecidas. Es importante señalar que, el crecimiento de esta ciudad se dio sobre terrenos inundables y espejos de agua, a través de la construcción de defensas y terraplenes (Viand \& González, 2012).

El día 29 de abril de 2003, la crecida extraordinaria del río Salado provocó la muerte de 152 personas y 130.000 habitantes debieron ser evacuados, haciendo de esta inundación una de las más catastróficas de la historia argentina (Ullberg, 2013). Este suceso incorporó a la gestión del riesgo, en la agenda de planificación urbana y en el año 2009, tuvo lugar la adhesión a la campaña de la Oficina de las Naciones Unidas para Reducción de Riesgo de Desastres y UNISDR para el desarrollo de ciudades resilientes.

Si bien tampoco se menciona de forma explícita el concepto de servicios ecosistémicos, las medidas orientadas a fomentar la mitigación y regulación de la escorrentía, precisamente se basan en la recuperación de espacios verdes y frentes fluviales naturales. Asimismo, es importante aclarar que, en el caso de esta ciudad santafecina, estas disposiciones de nivel local deben articularse con la Ley provincial 11730/00, que regula el régimen de uso de bienes situados en las áreas inundables. 


\section{Tabla 1. Zonificaciones que revalorizan espacios fluviales y ribereños en ciudades capitales de la Argentina.}

\begin{tabular}{|c|c|}
\hline Definición de las áreas o zonas delimitadas & $\begin{array}{l}\text { Ciudad e instrumento de } \\
\text { planificación que lo incluye }\end{array}$ \\
\hline $\begin{array}{l}\text { Distrito Parque Urbano Lagunar: Comprende los valles } \\
\text { de inundación de distintos cursos de agua, atendiendo a las } \\
\text { funciones de regulación hídrica que poseen como reservo- } \\
\text { rio de amortiguamiento de lluvias }\end{array}$ & Formosa $\left(\mathrm{OM} \mathrm{N}^{\circ} 6959 / 16\right)$ \\
\hline $\begin{array}{l}\text { Áreas especiales- Áreas ribereñas: Extensiones de tierra } \\
\text { urbana a proteger donde se establece la relación de borde } \\
\text { entre la ciudad y los entornos fluviales. }\end{array}$ & \multirow{4}{*}{ Santa Fe $\left(\mathrm{OM} \mathrm{N} \mathrm{N}^{\circ} 11748 / 10\right)$} \\
\hline $\begin{array}{l}\text { Áreas especiales- Cordones verdes: Extensiones de tierra } \\
\text { urbana con suelos absorbentes o pavimentos permeables } \\
\text { y población vegetal consistente. Incluyen espacios verdes } \\
\text { públicos, tierras libres y áreas vacantes públicas y privadas } \\
\text { y corredores ferroviarios. }\end{array}$ & \\
\hline $\begin{array}{l}\text { Distrito de seguridad hídrica (ZSH): Áreas no urbanizables } \\
\text { destinadas a obras estructurales de carácter hidrológicas sin } \\
\text { uso residencial de ningún tipo. }\end{array}$ & \\
\hline $\begin{array}{l}\text { Distrito EI-Especial de islas y frentes fluviales: Zonas } \\
\text { de islas y frentes fluviales de cursos permanentes de agua, } \\
\text { donde los agregados edilicios están subordinados a las } \\
\text { condiciones hídricas y ambientales. }\end{array}$ & \\
\hline $\begin{array}{l}\text { Costa Natural Urbana - Sector Arroyo Zaimán: espacios } \\
\text { verdes en ambas márgenes con el objeto de reconstruir su } \\
\text { equilibrio ecológico }\end{array}$ & Posadas (OM No 3372/13) \\
\hline $\begin{array}{l}\text { Zona Natural de Amortiguación: área de vinculación del } \\
\text { ecosistema de piedemonte con el área urbanizada. Brinda } \\
\text { servicios ambientales, como la reducción de la amenaza } \\
\text { aluvional por su cobertura vegetal. }\end{array}$ & \multirow[b]{2}{*}{ Mendoza (OM No 3944/18) } \\
\hline $\begin{array}{l}\text { Área de seguridad hídrica: Área donde la irregularidad de } \\
\text { precipitaciones y su concentración en el territorio generan } \\
\text { una red de cauces interconectados que ponen en peligro } \\
\text { aluvional a todo el sector. La vegetación se considera de } \\
\text { máxima importancia en este sector. }\end{array}$ & \\
\hline $\begin{array}{l}\text { Áreas de arroyos y bañados: Sector anegable del área ur- } \\
\text { bana. En parcelas superiores a } 300 \mathrm{~m}^{2} \text {, solo el } 40 \% \text { podrá } \\
\text { estar impermeabilizada. }\end{array}$ & La Plata (OM No 10703/10) \\
\hline
\end{tabular}

Fuente: Elaboración propia.

\section{Integración de infraestructura verde e indicadores urbanísticos en favor de la regulación hídrica}

Si se analiza el tipo de espacio que es alcanzado por esta normativa, en términos de su carácter público o privado, del total de las medidas 
halladas el $83 \%$ reglan sobre el espacio privado. Mientras que, en el espacio público las medidas se relacionan principalmente, con la promoción de cintas verdes, distritos de zonificación y áreas especiales, en los terrenos particulares la compensación de la pérdida de infiltración del suelo se busca a través de indicadores urbanísticos e intervenciones que impliquen principalmente, infraestructura verde o materiales porosos.

Asimismo, se identifica en ciudades como Corrientes, Resistencia y Ciudad Autónoma de Buenos Aires (CABA), la aplicación de un enfoque mixto que implica la integración de medidas de tipo infraestructural (Tabla 2) con medidas no estructurales plasmadas en normativa (Tabla 3). Tanto las reglamentaciones como la promoción del uso de materiales permeables, se alinean bajo dos claros objetivos, tales como la parquización de espacios libres y el límite a la impermeabilización del suelo urbano. A su vez, el objeto de aplicación es principalmente a escala de parcela diferenciándose de la zonificación que abarca distintas áreas de la ciudad.

Dentro de las intervenciones propuestas, se encuentra el uso de sistemas de captación y recuperación del agua de lluvia como también el uso de superficies vegetadas o porosas en los espacios libres. La vegetación no solo que se promueve en espacios peatonales o playas de estacionamientos, sino que en concordancia con una tendencia global su presencia se fomenta en terrazas, azoteas, techos, etc.

En el caso de estos últimos, es importante aclarar que el grado de contribución al servicio de mitigación y regulación de la escorrentía, dependerá de las especificaciones de su diseño. En la CABA, por ejemplo, se establece un espesor de suelo máximo para los techos verdes de $18 \mathrm{~cm}$. Si se tiene en cuenta que uno de los factores que influyen sobre el servicio ecosistémico de regulación hídrica es la profundidad del perfil de suelo, en este caso la profundidad limitada, podría dar lugar a un bajo potencial de retención de precipitaciones. En cualquier caso, la escorrentía generada sería menor a la producida por un espacio completamente impermeabilizado y nuevamente, resulta necesaria una mirada integral, debido a los múltiples servicios ecosistémicos, que provienen de estas áreas. 


\section{Tabla 2. Descripción de las medidas de infraestructura verde e intervenciones según objetivo de implementación}

\begin{tabular}{|c|c|c|}
\hline Objetivo & Descripción general & $\begin{array}{l}\text { Ciudad e instrumento de } \\
\text { planificación que lo incluye }\end{array}$ \\
\hline $\begin{array}{l}\text { Límite a la imper- } \\
\text { meabi-lización del } \\
\text { suelo urbano }\end{array}$ & $\begin{array}{l}\text { Exigencias para playas de estaciona- } \\
\text { miento de supermercados: contarán } \\
\text { con pavimento flexible, articulado o } \\
\text { retícula permeable y arbolado }\end{array}$ & Santa Rosa (OM No 3274/2005) \\
\hline \multirow{3}{*}{$\begin{array}{l}\text { Promoción de espa- } \\
\text { cios vegetados/ } \\
\text { parquizados }\end{array}$} & $\begin{array}{l}\text { Veredas con espacios verdes/aceras } \\
\text { con franja verde/cintas verdes: Vere- } \\
\text { das con senda peatonal embaldosada } \\
\text { de una dimensión dada; dejando el } \\
\text { resto como franja verde de césped. }\end{array}$ & $\begin{array}{l}\text { Resistencia (OM No 12926/18), } \\
\text { La Rioja OM No 1784/89), San- } \\
\text { tiago del Estero (Cód. de planea- } \\
\text { miento urbano y edificación), San } \\
\text { Juan (Cód. de Edificación), Vied- } \\
\text { ma (Cód. de edificación), Santa } \\
\text { Fe (OM No 11610/09). }\end{array}$ \\
\hline & $\begin{array}{l}\text { Terraza, azotea, balcón, cubierta } \\
\text { verde o ecológica: cubierta de techo } \\
\text { o terrazas, resuelta mediante trata- } \\
\text { miento con vegetación. }\end{array}$ & 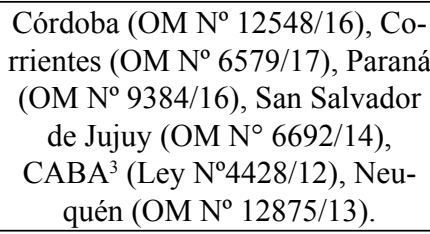 \\
\hline & $\begin{array}{l}\text { Muros y jardines verticales verdes: } \\
\text { Superficie total o parcial de los } \\
\text { muros externos de un edificio con } \\
\text { desarrollo de especies vegetales, } \\
\text { cubriendo dichos muros en forma } \\
\text { vertical. } \\
\end{array}$ & Paraná (Cód. de edificación). \\
\hline $\begin{array}{l}\text { Reducción de vo- } \\
\text { lumen de aguas } \\
\quad \text { pluviales }\end{array}$ & $\begin{array}{l}\text { Retardadores Pluviales/Sistema de } \\
\text { Recolección de Aguas de Lluvia/Su- } \\
\text { perficie de Captación Pluvial/Factor } \\
\text { de Captación/Sistema de ralenti- } \\
\text { zación de agua de lluvia captada/ } \\
\text { impacto hidrológico cero: superficie } \\
\text { que puede derivar las aguas pluviales } \\
\text { a un sistema de reutilización o para } \\
\text { su posterior derivación a sistemas de } \\
\text { desagües existentes. }\end{array}$ & $\begin{array}{l}\text { Paraná4 (Cód. de edificación y } \\
\left.\text { OMN }^{\circ} 9606 / 17\right) \text {, San Salvador } \\
\text { de Jujuy (OM N } 6702 / 14) \text {, Men- } \\
\text { doza (OM Nº 3944/18), CABA } \\
\text { (Cód. de Planeamiento Urbano), } \\
\text { Resistencia (OM Nº775/2007) }\end{array}$ \\
\hline
\end{tabular}

Fuente: Elaboración propia.

3 Limita a $18 \mathrm{~cm}$ el espesor máximo de suelo para el soporte de la vegetación.

4 Capacidad de reserva del sistema de captación en función del Riesgo Hídrico asociado a la localización del inmueble. 
Por otra parte, con los indicadores urbanísticos se intenta brindar una herramienta que permita calcular las superficies que debieran quedar libres de toda edificación y/o pavimento que impida o dificulte la infiltración de agua -Factor de impermeabilización del suelo (FIS) y sus variantes, como el Índice de permeabilidad, Superficie de suelo absorbente o Superficie absorbente- (Tabla 3).

Tabla 3. Indicadores urbanísticos para limitar la impermeabilización y fomentar la parquización de espacios libres

\begin{tabular}{|c|c|c|}
\hline Objetivo & $\begin{array}{c}\text { Denominación y } \\
\text { Descripción general }\end{array}$ & $\begin{array}{c}\text { Ciudad e instrumento de } \\
\text { planificación que lo incluye }\end{array}$ \\
\hline \multirow{3}{*}{$\begin{array}{l}\text { Límite a la imper- } \\
\text { meabilización del } \\
\text { suelo urbano }\end{array}$} & $\begin{array}{l}\text { Factor de ocupación del suelo (FOS): valor } \\
\text { máximo de ocupación de la masa edificada } \\
\text { en el predio. El resto del predio quedará libre } \\
\text { de toda edificación y deberá tener un trata- } \\
\text { miento permeable }\end{array}$ & $\begin{array}{c}\text { Resistencia (OM } \\
\left.\mathrm{N}^{\circ} 12926 / 18\right) \\
\text { Corrientes (Cód. de planea- } \\
\text { miento urbano) } \\
\text { Formosa }\left(\mathrm{OM} \mathrm{N}^{\circ} 6959 / 16\right) \text {. }\end{array}$ \\
\hline & $\begin{array}{l}\text { Factor de impermeabilización del suelo } \\
\text { (FIS)/Índice de permeabilidad/Superficie } \\
\text { de suelo absorbente/ Superficie absorbente } \\
\text { obligatoria: porcentaje de la superficie total } \\
\text { del lote que debe quedar libre de toda edifica- } \\
\text { ción y/o pavimento que impida y/o dificulte } \\
\text { la permeabilidad o infiltración de agua en el } \\
\text { suelo natural. }\end{array}$ & $\begin{array}{c}\text { Resistencia (OM No } \\
\text { 12926/18), Corrientes }{ }^{5} \text { (Res. } \\
\text { No }^{\circ} \text { 1465/19), San Salvador de } \\
\text { Jujuy (OM No 6702/14), Po- } \\
\text { sadas (OM N } 3372 / 13) \text {, Río } \\
\text { Grande (OM No 2863/11), } \\
\text { La Plata (OM No 10703/10), } \\
\text { CABA (Cód. Urbanístico), } \\
\text { Santa Fe (OM No 11748/10), } \\
\text { Córdoba (OM No 12596/16). }\end{array}$ \\
\hline & $\begin{array}{l}\text { Factor de impermeabilización total (FIT): } \\
\text { grado de impermeabilización o superficie } \\
\text { no absorbente total. Resulta de dividir la } \\
\text { superficie total construida más la superficie } \\
\text { de pisos no cubiertos, por la superficie total } \\
\text { del terreno. }\end{array}$ & $\begin{array}{c}\text { Resistencia (OM No } \\
12926 / 18) \text {, Corrientes (Res. } \\
\left.\mathrm{N}^{\mathrm{o}} 1465 / 19\right)\end{array}$ \\
\hline \multirow{2}{*}{$\begin{array}{l}\text { Promoción de es- } \\
\text { pacios vegetados/ } \\
\quad \text { parquizados }\end{array}$} & $\begin{array}{l}\text { Retiro de edificación: retiros (frente, fondo o } \\
\text { laterales) destinados a espacio verde }\end{array}$ & $\begin{array}{c}\text { San Fernando del Valle de } \\
\text { Catamarca (OM No 887/77), } \\
\text { Viedma (OM No 3319/96), } \\
\text { San Luis (OM No 968/77). }\end{array}$ \\
\hline & $\begin{array}{l}\text { Centro libre de manzana: superficie no edi- } \\
\text { ficable del terreno, destinada a espacio libre } \\
\text { verde y suelo absorbente, comprendida entre } \\
\text { las líneas de frente interno }\end{array}$ & $\begin{array}{c}\text { CABA (Código Urbanístico), } \\
\text { Posadas (OM No3372/13), } \\
\text { Mendoza (OM N³944/18), } \\
\text { Corrientes (Cód. de Planea- } \\
\text { miento Urbano). }\end{array}$ \\
\hline
\end{tabular}

Fuente: Elaboración propia.

5 Aplicado a un sector del área abarcada por el Plan Urbano Costero. 
Entre estas ciudades mencionadas, se destaca el caso de la capital de la provincia del Chaco, ciudad de Resistencia, en donde a través de la Ordenanza Municipal No 8775/2007, se busca el "impacto hidrológico cero" de las nuevas edificaciones sobre el sistema de drenaje existente. Es importante aclarar a su vez, que esta aglomeración urbana se dispone sobre la llanura de inundación del río Paraná, ocupando también las riberas de sus brazos y afluentes. Motivo por el cual, se han dado reiterados episodios de inundaciones que afectaron a un número considerable de habitantes. El crecimiento urbano sobre sectores inundables, llevó a la implementación de restricciones de uso del suelo, en función de las resoluciones de la Administración Provincial del Agua (APA) (Rohrmann \& Schaller, 2016).

Conjuntamente con estas zonificaciones, se establecieron medidas para el control de los cambios hidrológicos, que implican las nuevas construcciones. De esta manera, las propuestas de edificación deben acompañarse de una evaluación hidrológica, en la que el pico del hidrograma generado para un determinado Factor de Impermeabilización del Suelo (FIS) y Factor de impermeabilización total (FIT), no supere el pico que se obtiene bajo las características previas a la urbanización.

Para ello, se deberán prever dentro de cada parcela, dispositivos o mecanismos atenuantes del efecto producido por las precipitaciones intensas. Si bien, se trata de un avance importante en lo que respecta a medidas que regulen la impermeabilización, para su efectividad, también es necesaria una concordancia con los permisos de edificabilidad y los factores de ocupación del suelo. Precisamente, es a partir de estos parámetros que se define la posibilidad de mantener la forestación en los centros de las parcelas o la forestación de las aceras (Alcalá, 2012).

\section{Discusión \\ Cuantificación del servicio para contribuir con su incorporación en la normativa}

Si se indaga más allá del rango de ciudades objeto de estudio, se identifica que también en centros urbanos de menor jerarquía se está dando un proceso de incorporación de normativas, asociadas con prácticas de infiltración del agua de lluvia y restricción de la impermeabilización. Por ejemplo, en la ciudad de Toay (provincia de La Pampa) se ha impulsado la Ordenanza Municipal $N^{\circ}$ 5/14, que limita la 
impermeabilización de grandes superficies -como playas de estacionamiento o áreas de circulación-, para favorecer la recarga del acuífero, del cual se sustenta esta localidad.

Cabe aclarar que, las condiciones de semiaridez que caracterizan al clima de la región, refuerzan la necesidad de optimizar el manejo del recurso hídrico. Otro ejemplo es el proyecto de ordenanza en la ciudad costera de Mar del Plata (provincia de Buenos Aires), que se orienta a "la creación de un sistema de transporte permeable que corra paralelo a caminos, tales como cunetas verdes y franjas filtrantes, y pavimentos filtrantes" y "la implementación de un sistema de infiltración o control de agua, en origen en las zonas de media y baja densidad". En este caso, uno de los motivos que impulsa la generación de estas prácticas, es la problemática de la red de drenaje y la contaminación del frente costero, dado que el cuerpo receptor de los efluentes del sistema de desagües pluviales es el mar.

En este marco, la cuantificación y el mapeo de servicios ecosistémicos asociados a los cambios en el uso del suelo son fundamentales para hacer efectiva la contribución con la planificación y gestión territorial (Chen et al., 2020). En esta línea, Paruelo, Alcaraz-Segura, \& Volante (2011), señalan que la implementación de planes de manejo y ordenamiento del territorio requiere del seguimiento de los cambios en el nivel de provisión de los servicios ecosistémicos, con una cobertura completa del territorio. Estos autores sostienen que, para la toma de decisiones en cuestiones ambientales no solo es importante el inventario de los servicios ecosistémicos, sino también de la estimación de la tasa de provisión y demanda de los mismos considerando las fluctuaciones provocadas por las actividades antrópicas.

Las disposiciones examinadas, dan muestra de un inicio en la valorización de la regulación de escorrentía como servicio que pueden brindar los ecosistemas del entorno urbano. Sin embargo, más allá de que en la mayoría de los casos no existe una referencia explícita, restan aún especificaciones técnicas que permitan su cuantificación del servicio y por lo tanto, su incorporación en los instrumentos de planificación.

Existen, en la Argentina y el mundo, antecedentes en los que se aplican parámetros hidrológicos como indicadores de infiltración que podrían utilizarse para la cuantificación del servicio de regulación hídrica en áreas urbanas. Gittleman, Farmer, Kremer y McPhearson (2017), estimaron las 
tasas de absorción de escorrentía de aguas pluviales de los jardines comunitarios de la ciudad de Nueva York.

En Argentina, Zimmerman, Bracalenti y Onocko (2015), estimaron un indicador, basado en el coeficiente de escorrentía del método racional, que permitió cuantificar la magnitud del impacto de áreas verdes en el escurrimiento de subcuencas de la ciudad de Rosario. Detectaron que aumentos adecuados de superficie con cobertura vegetal, reducen significativamente el riesgo de inundaciones en los sistemas urbanos.

También es importante mencionar, la propuesta del protocolo ECOSER (Laterra, Castellarini \& Orúe, 2011), la cual propone como indicador de regulación hídrica, la proporción de agua de lluvia infiltrada frente a tormentas modales o con una recurrencia dada, a través del método de la curva-número del Soil Conservation Service. Por último, cabe destacar la propuesta Suárez y Alba (2017), en la que establecen indicadores de provisión y de demanda para la evaluación del impacto de las políticas locales sobre los servicios ecosistémicos en la ciudad de Madrid. Para el caso del servicio de regulación de la escorrentía superficial, proponen la capacidad de infiltración del suelo, el porcentaje de suelo impermeabilizado respecto al permeable, la reducción de escorrentía superficial y el porcentaje de zonas verdes, en áreas con riesgo de inundación como indicadores de provisión.

La valoración económica y social como guía de la implementación

\section{del enfoque ecosistémico en la desarrollo urbano}

Para dar contexto a estas consideraciones, es importante aclarar que, desde el ámbito legal, la figura de los servicios ecosistémicos no ha sido regulada en las jurisdicciones nacional, provincial y municipal (Ferro \& Minaverry, 2019). Si bien el concepto de servicios ecosistémicos es adoptado en la Ley 26331/07 de Presupuestos Mínimos de Protección Ambiental de los Bosques Nativos, el enfoque de los servicios ecosistémicos, dista aún de una incorporación plena en la jurisprudencia argentina.

Minaverry (2019), relaciona la escasa implementación de este concepto en la legislación, con la imposibilidad de capturar en su totalidad a los servicios ecosistémicos dentro del ámbito de los mercados comerciales. Esto último, se plantea como un punto fundamental en la discusión, en torno a la monetización de los servicios ecosistémicos como forma de valoración. 
Para el caso del servicio de regulación hídrica y mitigación de la escorrentía, el valor está dado por el proceso y no por la generación de un bien. En la práctica el hecho de priorizar este servicio, puede traducirse en compensaciones económicas en términos de subsidios o beneficios tributarios para quienes prioricen su funcionamiento, a través del seguimiento de la normativa o de la incorporación de determinados tipos de cobertura del suelo. Para ello, además de la cuantificación del servicio es también imprescindible una valoración social.

Esto último, es esencial para el proceso de construcción de consenso, pues guía su priorización y permite la generación de distintos escenarios para el crecimiento de la ciudad (Paruelo et al., 2011). Sobre este asunto, resulta clave cuantificar el nivel de apropiación de beneficios y perjuicios, por parte de los distintos actores socioeconómicos.

Avendaño-Leadem, Cedeño-Montoya y Arroyo-Zeledón (2020), concuerdan en que reconocer explícitamente el valor del servicio ecosistémico como referencia dentro de la ordenación territorial contribuye con el balance de las disyuntivas en torno al uso de la tierra y los múltiples escenarios de desarrollo. Para ello, es importante que en los planes se describan las compensaciones o pérdidas que se presentan entre los diferentes usos y servicios de la tierra y cómo evaluarlas.

De esta manera, la combinación del conocimiento cualitativo y cuantitativo acerca de las dinámicas ecosistémicas y de la distribución espacial y temporal del nivel de provisión de los servicios ecosistémicos, puede traducirse en herramientas de planificación urbana y territorial.

En esta línea, es creciente el número de estudios que abordan los beneficios monetarios asociados con los servicios ambientales de los espacios verdes urbanos, principalmente aquellos relacionados con la gestión de la escorrentía urbana. Ejemplo de ello, son los estudios realizados en ciudades de China y Finlandia (Zhang, Xie, Zhang \& Zhang, 2012; Silvennoinen, et al., 2017) en los que a través de métodos de valuación por reemplazo, se comparó el costo del tendido y mantenimiento de los sistemas de desagües pluviales con el gasto que implica el cuidado de parques y áreas verdes. Estos cálculos a su vez, se complementaron con la modelización de la escorrentía sobre coberturas con distintos grados de impermeabilización y sobre áreas sin construir. Mostrando un claro aumento en el primer caso, lo que contribuye a su vez con un aumento en el costo. 
Otro aspecto fundamental para la incorporación efectiva del paradigma ecosistémico en los instrumentos de planificación urbana, es la correspondencia entre los aspectos que hacen a la provisión de los servicios, con una institucionalidad sólida sobre la que se apoyen las normativas.

Tal como lo expresa Minaverry (2019), se requiere de un marco que permita establecer políticas, normas y protocolos, según la comprensión científica de las interacciones y procesos ecológicos necesarios para mantener la estructura, función y dinámicas de los ecosistemas involucrados. De esta manera, las prácticas de mitigación o compensaciones de la urbanización, deben apoyarse en un sistema común de conocimiento basado en los usos sostenibles de la tierra (Giaimo, Regis \& Salata, 2016).

Los esfuerzos para actualizar las infraestructuras urbanas frente a las presiones del cambio climático junto con los cambios demográficos, requieren enfoques holísticos que incorporen a la naturaleza como parte de la ecuación (Frantzeskaki, 2019). Ello significa el replanteamiento radical del sistema de gobernanza local y en particular, de la actividad de planificación como instrumento tanto normativo como estratégico (Giaimo et al., 2016). En el caso argentino, un cambio de este tenor se plantea como un escenario lejano. No obstante, los zonificaciones e indicadores identificados dan indicios de una valorización en el rol de los servicios ecosistémicos, que derivan del ecosistema urbano.

\section{Conclusión}

El $87,5 \%$ de las ciudades analizadas, presentan algún tipo de medida urbanística que se relaciona con los factores asociados al servicio ecosistémico de regulación hídrica o con la gestión de aguas pluviales, desde una perspectiva distinta a la convencional. Sin embargo, en la mayoría de los casos no existe una referencia explícita al enfoque ecosistémico, lo que dificulta la cuantificación en su nivel de provisión servicio y por lo tanto, su incorporación en los instrumentos de planificación.

Por otra parte, el análisis focalizado en el tipo de medidas reglamentadas permitió identificar que, las zonificaciones se orientan a los componentes del ecosistema urbano, a través de la preservación de áreas ribereñas y lagunares. Mientras que, las referencias a la capacidad de infiltración se dan en los indicadores urbanísticos, que buscan calcular las superficies que debieran quedar libres para contribuir con esta función hidrológica. 
Paralelamente, se han identificado normativas que alientan la construcción de infraestructura verde y el uso de materiales porosos. Estas iniciativas, muestran en los instrumentos de planificación urbana de la capital nacional y las capitales provinciales de la Argentina, un reconocimiento del impacto hidrológico de la urbanización. Su compensación se busca a escala de terrenos particulares con indicadores, que limitan la impermeabilización, mientras que en el espacio público las medidas implementadas se relacionan principalmente con la promoción de cintas verdes, distritos de zonificación y áreas especiales.

Asimismo, existe una preponderancia por parte de las ciudades asentadas en los valles de los grandes ríos de la cuenca del Plata. Sin embargo, la valorización de la regulación de la escorrentía junto con la implementación de infraestructura verde, está ganando terreno como alternativa de conservación y almacenamiento de agua en entornos urbanos de clima semiárido.

Del análisis realizado puede concluirse que, el enfoque de los servicios ecosistémicos encuentra puntos de aplicación en la normativa de planificación urbana. De esta manera, se propone la continuación de estudios en materia de servicios ecosistémicos urbanos, para su incorporación en los instrumentos de planificación de la ciudad, contribuyendo al mejoramiento de la calidad ambiental de la misma.

Esto supone la necesidad de superar obstáculos que están dados por la ambigüedad de las definiciones de los servicios de los ecosistemas, la variedad de puntos de vista sobre los activos ambientales en la sociedad, los problemas de escala y la multiplicidad de métodos para la evaluación de los servicios de los ecosistemas (Kowalczyk \& Sudra, 2014).

A su vez, para el caso específico de la Argentina, el avance sobre la cuantificación del nivel de provisión y la valorización por parte de los diferentes actores sociales del espacio urbano, son aspectos que podrían marcar el inicio de una incorporación efectiva en los instrumentos de planificación urbana.

En un marco de crecimiento extensivo de las ciudades que implica el consumo y modificación del suelo, contar con prácticas de mitigación se vuelve fundamental para contrarrestar los efectos adversos que pasen a ser desafíos para la gestión. Es vigente, el interés de la investigación científica sobre el fundamento socio-ecológico de los servicios ecosistémicos y el establecimiento de la influencia de las acciones humanas. Por lo que, el entendimiento de la incorporación de este conocimiento en la toma de decisiones, se plantea como el próximo paso a seguir. 


\section{Agradecimientos}

Las autoras agradecen los comentarios y sugerencias de los revisores/a que contribuyeron con la mejora del artículo. El trabajo se realizó en el marco del PGI "Geografía Física aplicada al estudio de la interacción sociedad-naturaleza. Problemáticas a diversas escalas témporo-espaciales" (24/ZG78), financiado por la SGCyT (UNS).

\section{Referencias}

Ahern, J., Cilliers, S., \& Niemelä, J. (2014). The concept of ecosystem services in adaptive urban planning and design: A framework for supporting innovation. Landscape and Urban Planning, 125, 254-259.

Alcalá, L. I. (agosto, 2012). Avatares de la manzana. Entre la dispersión y la densificación. En: IX Bienal del Coloquio de Transformaciones Territoriales. Universidad Nacional de Tucumán, Argentina.

Altesor, A., Barral, M. P., Booman, G., Carreño, L., Cristeche, E., Isacch, J. P., Maceira, N., \& Pérez, N. (2011). Servicios ecosistémicos: un marco conceptual en construcción. Aspectos conceptuales y operativos. En P. Laterra, E. Jobbágy \& J. Paruelo, (Eds.). Valoración de Servicios Ecosistémicos: conceptos, herramientas y aplicaciones para el ordenamiento territorial (pp. 645-657). Buenos Aires: Ediciones INTA.

Argañaraz, J. P., \& Lorenz, G. (2010). Contribución de las áreas verdes urbanas a la regulación del balance de agua en Santiago del Estero, Argentina. Bosque (Valdivia), 31(3), 231-242.

Avendaño-Leadem, D., Cedeño-Montoya, B., \& Arroyo-Zeledón, M. S. (2020). Integrando el concepto de servicios ecosistémicos en el ordenamiento territorial. Revista Geográfica de América Central. 2 (65), 63-90.

Balvanera, P., Uriarte, M., Almeida-Leñero, L., Altesor, A., DeClerck, F., Gardner, T., ... \& Vallejos, M. (2012). Ecosystem services research in Latin America: The state of the art. Ecosystem Services, 2, 56-70.

Barros, V. R., Boninsegna, J. A., Camilloni, I. A., Chidiak, M., Magrín, G. O., \& Rusticucci, M. (2015). Climate change in Argentina: trends, projections, impacts and adaptation. Wiley Interdisciplinary Reviews: Climate Change, 6(2), 151-169. 
Bolund, P., \& Hunhammar, S. (1999). Ecosystem services in urban areas. Ecological economics. 29(2), 293-301.

Laterra, P., Castellarini, F., \& Orúe, M. E. (2011). ECOSER: Un protocolo para la evaluación biofísica de servicios ecosistémicos y la integración con su valor social. En P. Laterra, E. Jobbágy, \& J. Paruelo, (Eds.). Valoración de Servicios ecosistémicos: Conceptos, herramientas y aplicaciones para el Ordenamiento Territorial (pp. 35989). Buenos Aires: Ediciones INTA.

Chen, D., Li, J., Yang, X., Zhou, Z., Pan, Y., \& Li, M. (2020). Quantifying water provision service supply, demand and spatial flow for land use optimization: A case study in the YanHe watershed. Ecosystem Services, 43, 101-117.

Daily, G. (1997). Nature's services: societal dependence of ecosystems. Washington, D. C.: Island Press.

Ferro, M., \& Minaverry, C. M. (2019). Aportes normativos, institucionales y sociales a la gestión del agua y el enfoque ecosistémico en la Cuenca del Río Luján, Argentina. Revista de Derecho (20), 25-55. Recuperado de: http://www.scielo.edu.uy/scielo. php?pid=S2393-61932019000200025\&script=sci_arttext

Frantzeskaki, N. (2019). Seven lessons for planning nature-based solutions in cities. Environmental science \& Policy, 93, 101-111.

Giaimo, C., Regis, D., \& Salata, S. (2016). Ecosystem Services and Urban Planning. Tools, Methods and Experiences for an Integrated and sustainable territorial government. En Sustainable Buil Environment 2016 - "Towards post-carbon cities". Torino, Italia. Recuperado de: https://re.public.polimi.it/handle/11311/995171\#.X20f4cJKjIU

Gómez-Baggethun, E., \& Barton, D. N. (2013). Classifying and valuing ecosystem services for urban planning. Ecological Economics, 86, 235-245.

Gómez-Baggethun, E., Martín-López, B., Barton, D., Braat, L., Saarikoski, H., Kelemen, E., \& Potschin, M. (2014). State of the art report on integrated valuation of ecosystem services. EU FP7 OpenNESS Project Deliverable, 4, 1-33.

González, S., Torchia, N., \& Viand, J. (2015). Vulnerabilidad asociada a la ocupación de terrenos en áreas inundables. Cambio Climático e 
Inundaciones Urbanas Buenos Aires, Argentina: Secretaría de Ambiente y Desarrollo Sustentable de la Nación.

Grizzetti, B., Lanzanova, D., Liquete, C., Reynaud, A., \& Cardoso, A. C. (2016). Assessing water ecosystem services for water resource management. Environmental Science \& Policy, 61, 194-203.

Gittleman, M., Farmer, C. J., Kremer, P., \& McPhearson, T. (2017). Estimating stormwater runoff for community gardens in New York City. Urban ecosystems, 20(1), 129-139.

Honorable Congreso de la Nación Argentina (26 de diciembre 2007). Ley 26331. Ley de Protección ambiental de los bosques nativos. Boletín Oficial $N^{\circ} 31310$. República Argentina.

Honorable Congreso de la Nación Argentina (20 de noviembre 2019).

Ley 27520. Ley de Presupuestos mínimos de adaptación y mitigación al cambio climático global. Boletín Oficial $N^{\circ} 99081$. República Argentina.

Honorable Concejo Deliberante de Paraná (2018). Cuencas hidrográficas urbanas. Ordenanza 9668. Recuperado dehttp://190.183.231.163:4892/ digesto/spip.php?article6679

Jansson, Å. (2013). Reaching for a sustainable, resilient urban future using the lens of ecosystem services. Ecological Economics, 86, 285-291. Jullian, C., Nahuelhual, L., Mazzorana, B., \& Aguayo, M. (2018). Evaluación del servicio ecosistémico de regulación hídrica ante escenarios de conservación de vegetación nativa y expansión de plantaciones forestales en el centro-sur de Chile. Bosque (Valdivia), 39(2), 277-289.

Kowalczyk, M., \& Sudra, P. (2014). Ecosystem services in spatial planning. Europa XXI, 27, 5-18. doi: https://doi.org/10.7163/eu21.2014.27.1

Ley Provincial 11730. (2000). Bienes zonas inundables- Inundaciones. Boletín Oficial Santa Fe. República Argentina. Recuperado de https://www.santafe.gov.ar/index.php/web/content/download/128926/637160/file/Ley\%20Provincial\%2011730\%20-\%20 Bienes\%20zonas\%20inundables.pdf

Maragno, D., Gaglio, M., Robbi, M., Appiotti, F., Fano, E. A. \& Gissi, E. (2018). Fine-scale analysis of urban flooding reduction from green infrastructure: An ecosystem services approach for the management of water flows. Ecological Modelling, 386, 1-10. 
Millennium Ecosystem Assessment. (2005). Ecosystems and Human WellBeing: A Framework for Assessment. Washington, DC: Island Press.

Minaverry, C. M. (2019). Enfoque ecosistémico, pago de servicios y análisis comparativo del marco legal para la protección de los bosques nativos en dos regiones forestales argentinas. En Ius et Praxis, 25(1), 441-480. Organización de Naciones Unidas. (2015). Transformar nuestro mundo: la Agenda 2030 para el Desarrollo Sostenible. A/RES/70/1. Recuperado de: https://undocs.org/es/A/RES/70/1

Paruelo, J., Alcaraz-Segura, D. \& Volante, J. N. (2011). El seguimiento del nivel de provisión de los servicios ecosistémicos. En P.Laterra, E. Jobbágy, \& J. Paruelo, (Eds.). Valoración de Servicios ecosistémicos: Conceptos, herramientas y aplicaciones para el Ordenamiento Territorial (pp. 141-162). Buenos Aires: Ediciones INTA.

Pedersen, M. (2012). Ecosystem services analysis for the design of regenerative built environments. Building Research \& Information, 40(1), 54-64.

Pickett, S. T., Cadenasso, M. L., Grove, J. M., Nilon, C. H., Pouyat, R. V., Zipperer, W. C. \& Costanza, R. (2001). Urban ecological systems: linking terrestrial ecological, physical, and socioeconomic components of metropolitan areas. Annual review of ecology and systematics, 32(1), 127-157.

Rohrmann, H. y Schaller, O. (2016). Experiencias de 20 años en gestión de línea de ribera y zonificación de riesgo hídrico, Resistencia-Chaco. En Seminario Taller Línea de Ribera y Riesgo Hídrico. Facultad de Ingeniería y Ciencias Hídricas (FICH-UNLP). La Plata, Argentina.

Silvennoinen, S., Maija, T., Vesa Y., Harri K., Markku O. \& Heikki, S. (2017). Monetary Value of Urban Green Space as an Ecosystem Service Provider: A Case Study of Urban Runoff Management in Finland." Ecosystem Services, 28, 17-27. doi: https://doi.org/10.1016/j. ecoser.2017.09.013.

Secretaria de Ambiente y Desarrollo Sustentable. (2019). Protocolo: Ciudades Sustentables y Resilientes. Buenos Aires, Argentina.

Suárez, M., \& Alba, D. (2017). Evaluación del impacto de las políticas del Ayuntamiento de Madrid en losservicios de los ecosistemas (20032015). CONAMA Local Valencia. Recuperado de : http://www. conama11.vsf.es/conama10/download/files/conamalocal2017/CT 
Tan, P. Y., Zhang, J., Masoudi, M., Alemu, J. B., Edwards, P. J., GrêtRegamey, A., Richards, D. R., Song, X. P. \& Wong, L. W. (2020). A conceptual framework to untangle the concept of urban ecosystem services. Landscape and urban planning, 200, 103837.

Terraza, H., Rubio, D. \& Vera, F. (2016). De ciudades emergentes a ciudades sostenibles. Santiago de Chile: Ed. ARQ.

Ullberg, S. (2013). Watermarks: urban flooding and memoryscape in Argentina. Tesis Doctoral. Universidad de Estocolmo. Recuperado de: http:/www.diva-portal.org/smash/get/diva2:624415/FULLTEXT01.pdf

Viand, J. \& González, S. (2012). Crear riesgo, ocultar riesgo: gestión de inundaciones y política urbana en dos ciudades argentinas. En Primer Encuentro de Investigadores en Formación de Recursos Hidricos. Buenos Aires, Instituto Nacional del Agua. Recuperado de:http://repo. floodalliance.net/jspui/bitstream/44111/2323/1/01_027_Viand.pdf

Villarreal, E. L. \& Bengtsson, L. (2005). Response of a Sedum green-roof to individual rain events. Ecological Engineering, 25(1), 1-7.

Zhang, B., Xie, G.,Zhang, G., \& Zhang, J. (2012). The Economic Benefits of Rainwater-Runoff Reduction by Urban Green Spaces: A Case Study in Beijing, China.Journal of Environmental Management, 100, 65-71. doi: https://doi.org/10.1016/j.jenvman.2012.01.015.

Zimmermann, E., Bracalenti, L., \& Onocko, A. (2015). Infraestructura verde como alternativa sustentable para reducir el riesgo hídrico en áreas urbanizadas. Cuadernos del CURIHAM, (21), 55-69. 